\title{
Space-Mapping-Based Modeling Utilizing Parameter Extraction with Variable Weight Coefficients and a Data Base
}

\author{
Slawomir Koziel, Member, IEEE, and John W. Bandler, Fellow, IEEE \\ Department of Electrical and Computer Eng., McMaster University, Hamilton, ON, Canada L8S 4K1
}

\begin{abstract}
A new space-mapping-based surrogate modeling methodology is presented. We assume that certain fine model data, the so-called base set, is available in the region of interest. To evaluate the surrogate, we perform parameter extraction with weighting coefficients dependent on the distance between the point of interest and base points. This has advantages over standard SM modeling: (1) it can handle any base set, (2) the accuracy of the surrogate improves while the number of points in the base set grows even if the flexibility of the SM surrogate remains unchanged, (3) the model evaluation cost is roughly independent of the size of the base set. Examples confirm theoretical considerations and demonstrate robustness.
\end{abstract}

Index Terms-Computer-aided design (CAD), EM modeling, space mapping, surrogate modeling.

\section{INTRODUCTION}

Full-wave EM simulations of microwave structures are CPU intensive. Statistical analysis and yield optimization, crucial for manufacturability-driven designs in a time-tomarket development environment, demand accurate and fast models. The Space Mapping (SM) concept [1], [2] addresses this issue.

SM assumes "fine" and "coarse" models. The "fine" model may be a high fidelity CPU-intensive EM simulator, undesirable for direct statistical analysis and design. The "coarse" model can be a simplified representation such as an equivalent circuit with empirical formulas. SM modeling [3][5] exploits the speed of the coarse model and the accuracy of the fine model to develop fast, accurate enhanced models (surrogates) valid over a wide range of parameter values.

We present a new SM-based modeling methodology. The proposed method aims at overcoming limitations of the standard methodology which is based on setting up the surrogate model using a small amount of fine-model data (usually, $2 n+1$ points, where $n$ is the number of design variables) and performing extraction of model parameters over the whole set of this data [6], [7]. This is a simple

This work was supported in part by the Natural Sciences and Engineering Research Council of Canada under Grants OGP0007239 and STGP269760, and by Bandler Corporation.

S. Koziel is with the Simulation Optimization Systems Research Laboratory, Department of Electrical and Computer Engineering, McMaster University, Hamilton, ON, Canada L8S 4K1.

J.W. Bandler is with the Simulation Optimization Systems Research Laboratory, Department of Electrical and Computer Engineering, McMaster University, Hamilton, ON, Canada L8S 4K1 and also with Bandler Corporation, Dundas, ON, Canada L9H 5E7. methodology and gives reasonable accuracy especially for low-dimensional problems. In order to further improve the modeling performance one needs to involve more fine model information. Unfortunately, SM is not suitable to handle large a amount of fine model data by itself, i.e., increasing the number of base points does not help if the number of degrees of freedom of the model remains unchanged. Possible solutions are: (1) dividing the region of interest into smaller subregions so that a separate SM-model is set up for each subregion (problems: the number of regions grows exponentially with the dimension of the design parameter space; possible discontinuity at the border between the regions); (2) increasing the number of degrees of freedom of the SM model (problem: a large number of model parameters to be extracted). Another approach, the one we exploit here, is to evaluate SM surrogate models on-the-fly using parameter extraction with coordinate-dependent weighting coefficients.

\section{New SM MODELING MeTHODOLOGY}

Let $\boldsymbol{R}_{f}: X_{f} \rightarrow R^{m}$ and $\boldsymbol{R}_{c}: X_{c} \rightarrow R^{m}$ denote the fine and coarse model response vectors, where $X_{f} \subseteq R^{n}$ and $X_{c} \subseteq R^{n}$ are design variable domains of the fine and coarse models, respectively. For example, $\boldsymbol{R}_{\boldsymbol{f}}(\boldsymbol{x})$ and $\boldsymbol{R}_{c}(\boldsymbol{x})$ may represent the magnitude of a transfer function at $m$ chosen frequencies.

We denote by $X_{R} \subseteq X_{f}$ the region of interest in which we want enhanced matching between the surrogate and the fine model. We assume that $X_{R}$ is an $n$-dimensional interval in $R^{n}$ with center at reference point $\boldsymbol{x}^{0}=\left[\begin{array}{lll}x_{0.1} & \ldots & x_{0 . n}\end{array}\right]^{T} \in R^{n}$.

$$
X_{R}=\left[\boldsymbol{x}^{0}-\boldsymbol{\delta}, \boldsymbol{x}^{0}+\boldsymbol{\delta}\right]=\left[x_{0,1}-\delta_{1}, x_{0,1}+\delta_{1}\right] \times \ldots \times\left[x_{0, n}-\delta_{n}, x_{0, n}+\delta_{n}\right]
$$

where $\delta=\left[\begin{array}{lll}\delta_{1} & \ldots & \delta_{n}\end{array}\right]^{T}$ determines the size of $X_{R}$. We use $X_{R}\left(x^{0}, \delta\right)$ to denote the region of interest defined by $\boldsymbol{x}^{0}$ and $\delta$. Suppose we have the base set $X_{B}=\left\{\boldsymbol{x}^{1}, \boldsymbol{x}^{2}, \ldots, \boldsymbol{x}^{N}\right\} \subset X_{R}\left(\boldsymbol{x}^{0}, \boldsymbol{\delta}\right)$, where $N$ is the number of base points, such that the fine model response is known at all points $\boldsymbol{x}^{j}, j=1,2, \ldots, N$. We do not assume any particular location of the base points.

We define a generic surrogate model $\boldsymbol{R}_{s}: X_{f} \times M_{m \times m}$ $\times M_{n \times n} \times M_{n \times 1} \times M_{m \times 1} \rightarrow R^{m}$ as

$$
R_{s}(\boldsymbol{x}, \boldsymbol{A}, \boldsymbol{B}, \boldsymbol{c}, \boldsymbol{d})=\boldsymbol{A} \cdot \boldsymbol{R}_{c}(\boldsymbol{B} \cdot \boldsymbol{x}+\boldsymbol{c})+\boldsymbol{d}
$$

with matrices $\boldsymbol{A}=\operatorname{diag}\left\{a_{1}, \ldots, a_{m}\right\}, \quad \boldsymbol{B} \in M_{n \times n}, \quad \boldsymbol{c} \in M_{n \times 1}$, and $\boldsymbol{d} \in M_{m \times 1}\left(M_{k \times l}\right.$ denotes the set of $k \times l$ real matrices) found using the parameter extraction (PE) 


$$
\begin{gathered}
(\boldsymbol{A}, \boldsymbol{B}, \boldsymbol{c}, \boldsymbol{d})=\arg \min _{(\boldsymbol{\alpha}, \boldsymbol{\beta}, \boldsymbol{\gamma}, \boldsymbol{\delta})}\left\{\sum_{k=1}^{N} w_{k}(\boldsymbol{x})\left\|\boldsymbol{R}_{f}\left(\boldsymbol{x}^{k}\right)-\boldsymbol{R}_{s}\left(\boldsymbol{x}^{k}, \boldsymbol{\alpha}, \boldsymbol{\beta}, \boldsymbol{\gamma}, \boldsymbol{\delta}\right)\right\|\right. \\
\left.+\sum_{k=1}^{N} v_{k}(\boldsymbol{x})\left\|\boldsymbol{J}_{\boldsymbol{R}_{f}}\left(\boldsymbol{x}^{k}\right)-\boldsymbol{J}_{\boldsymbol{R}_{s}}\left(\boldsymbol{x}^{k}, \boldsymbol{\alpha}, \boldsymbol{\beta}, \boldsymbol{\gamma}, \boldsymbol{\delta}\right)\right\|\right\}
\end{gathered}
$$

Apart from model (2), (3), optional frequency scaling can be implemented that works in such a way that the coarse model is evaluated at a different frequency than the fine model using the transformation: $\omega \rightarrow f_{0}+f_{1} \omega$, where $\boldsymbol{F}=\left[f_{0} f_{1}\right] \in R^{2}$ is obtained together with matrices $A, B, c$, and $d$ using a parameter extraction process similar to (3).

By imposing constraints on the parameter extraction procedure, we can generate different models with different flexibility and number of degrees of freedom. Here, however, we use the full model as above.

Note that the weighting coefficients in (3) are functions of $\boldsymbol{x}$. In practice we use $v_{k} \equiv 0$ for $k=1,2, \ldots, N$. Coefficients $w_{k}$ are calculated according to

$$
w_{k}=w_{k}(\boldsymbol{x} ; C, \lambda)=\frac{\exp \left(-\frac{\left\|\boldsymbol{x}-\boldsymbol{x}^{k}\right\|^{2}}{C \cdot \lambda^{2}}\right)}{\sum_{j=1}^{N} \exp \left(-\frac{\left\|\boldsymbol{x}-\boldsymbol{x}^{j}\right\|^{2}}{C \cdot \lambda^{2}}\right)} \quad k=1,2, \ldots, N
$$

where $\boldsymbol{x}$ is the evaluation point, $\lambda=\lambda(\delta, N)$ is a characteristic distance depending on the size of the region of interest and the number of base points

$$
\lambda(\boldsymbol{\delta}, N)=\frac{2}{n N^{1 / n}} \sum_{i=1}^{n} \delta_{i}
$$

If the base points are uniformly distributed in $X_{R}, \lambda=\lambda(\delta, N)$ is just an average distance between neighbouring points. Constant $C>0$ determines how fast the weighting coefficients decrease with increase of base-point distance from $x$. We use $C=1$, a value found to give good performance.

In a practical implementation, especially if the dimension of the design variable space is larger, it is desirable to limit the number of base points included in the parameter extraction to make PE reasonably fast. We use a threshold for coefficients $w_{k}$ so that the coefficients are set to zero if their value is below this threshold (we use a relative threshold, i.e., a fraction of $w_{\max }=\max \left\{w_{k}: k=1,2, \ldots, N\right\}$; in our experiments we use $0.01 w_{\text {max }}$ ). In this way, we make evaluation of the matching condition function cheaper. On the other hand, using smaller values of $C$ allows us to reduce the number of base points because a smaller value of $C$ causes faster decrease of the coefficients $w_{k}$ while moving away from $\boldsymbol{x}$ so that only a few of them are above the aforementioned threshold. We also use hard limits for the number of points in parameter extraction. We use both minimum and maximum values: a maximum limit to reduce evaluation cost, a minimum limit to assure that the number of points is enough to maintain accuracy.

The standard SM modeling technique [6] can be obtained from (2)-(4) by choosing a standard (i.e., star-distribution-like) $X_{B}$ and letting $C \rightarrow \infty$ in (4).

It is intuitively obvious that modeling accuracy depends on characteristic distance $\lambda$, in particular, that accuracy improves with decreasing $\lambda$.

\section{EXAMPLES}

We consider a capacitively-loaded 10:1 impedance transformer. The "coarse" model and the "fine" model are an ideal two-section transmission line (TL) and a capacitivelyloaded TL with capacitors $C_{1}=C_{2}=C_{3}=10 \mathrm{pF}$ as shown in Fig. 1. The characteristic impedances are kept fixed at the optimal values $Z_{1}=4.4721 \Omega$ and $Z_{2}=2.2361 \Omega$. The electrical lengths $L_{1}$ and $L_{2}$ at $1 \mathrm{GHz}$ are chosen as design parameters. The frequency range is $0.5 \mathrm{GHz} \leq \omega \leq 1.5 \mathrm{GHz}$ with a step of

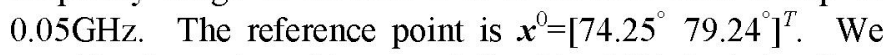
consider the region of interest defined by $10 \%$ deviation from $\boldsymbol{x}^{0}$. The fine and coarse models are implemented in Matlab. We consider the input reflection coefficient as model response.

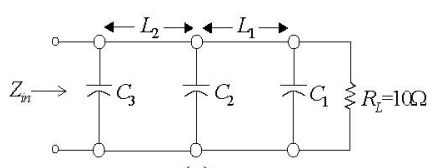

(a)

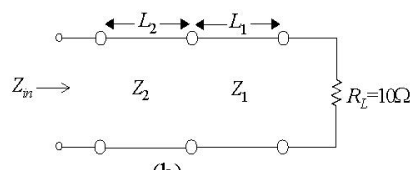

(b)
Fig.1. Fine (a) and coarse (b) model, two-section capacitively-loaded impedance transformer

We performed a number of experiments for this example using both the standard surrogate model and the new model (2), (3). Accuracy of the standard model was compared with accuracy of the new model for different base sets. Table I shows details of the base sets used in the experiments. Accuracy was tested using 30 test points randomly distributed in the region of interest. The error measure used was the $l_{2}$ norm of the difference between the fine model response and the corresponding surrogate model response.

TABLE I

Base Set Data For Two-Section TRansformer EXample

\begin{tabular}{cccc}
\hline \hline Base set & Description & Number of points & $\lambda$ \\
\hline$X_{B 1}$ & Uniform mesh $+\boldsymbol{x}^{0}$ & 5 & 6.86 \\
$X_{B 2}$ & Uniform mesh & 9 & 5.12 \\
$X_{B 3}$ & Uniform mesh & 25 & 3.07 \\
$X_{B 4}$ & Uniform mesh & 100 & 1.53 \\
\hline \hline
\end{tabular}

Table II shows numerical results (error statistics) for the standard model and the new surrogate with all the base sets considered. Figs. 2-4 show error plots (modulus of the difference between the fine model and the corresponding surrogate model response versus frequency) for the standard model and the new model with base sets $X_{B 3}$ and $X_{B 4}$, respectively. All the experiments were performed using constant $C$ equal to 1.0 (cf. (4)). Fig. 4 shows dependence of average modeling error on the characteristic distance $\lambda$. The results show that the new surrogate model performs as expected. Modeling accuracy improves while characteristic distance of the base set gets smaller. Note that the new surrogate model performs similarly as the standard model for base sets of the same cardinality as the star distribution-based set. The standard model is clearly outperformed by the new model if the base set is increased. 
TABLE II

MODELING RESUlTS FOR FOR TWO-SECTION TRANSFORMER EXAMPLE. VERIFICATION FOR 30 RANDOM TEST POINTS

\begin{tabular}{ccccc}
\hline \hline Model & Base set & $\begin{array}{c}\text { Average } \\
\text { Error }\end{array}$ & $\begin{array}{c}\text { Maximum } \\
\text { Error }\end{array}$ & $\begin{array}{c}\text { Standard } \\
\text { Deviation }\end{array}$ \\
\hline Standard & star dist. & 0.0389 & 0.0676 & 0.0152 \\
New & $X_{B 1}$ & 0.0384 & 0.0663 & 0.0153 \\
New & $X_{B 2}$ & 0.0218 & 0.0717 & 0.0142 \\
New & $X_{B 3}$ & 0.0073 & 0.0156 & 0.0038 \\
New & $X_{B 4}$ & 0.0027 & 0.0046 & 0.0013 \\
\hline \hline
\end{tabular}

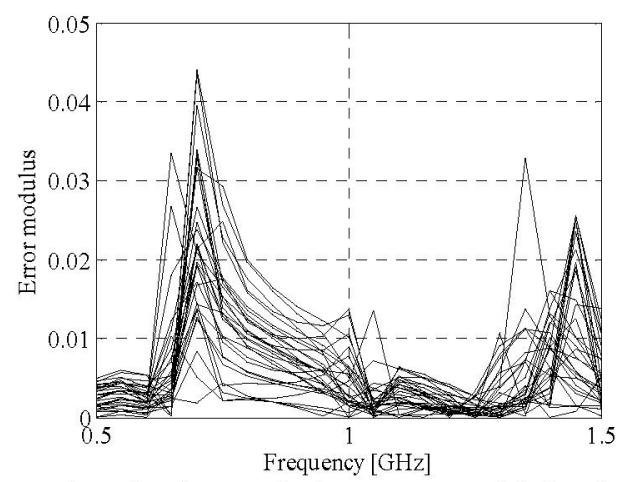

Fig.2. Error plots for the standard surrogate model for the two-section impedance transformer ( 30 test points).

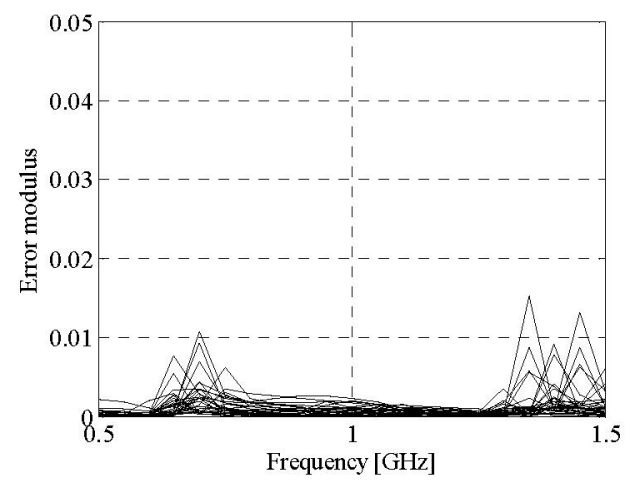

Fig.3. Error plots for the new surrogate model with base set $X_{B 3}$ for the two-section impedance transformer (30 test points).

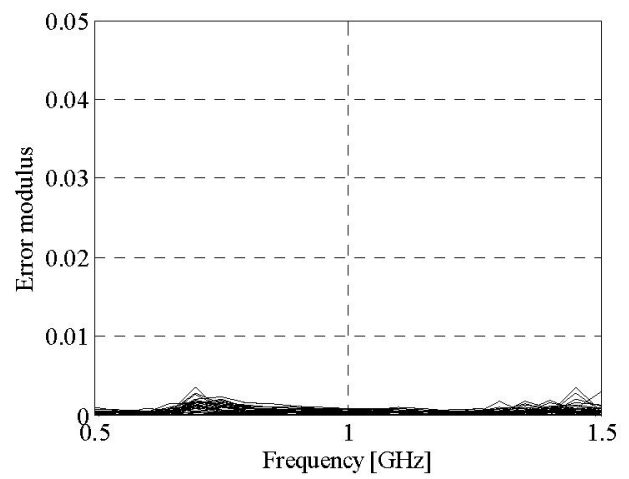

Fig.4. Error plots for the new surrogate model with base set $X_{B 4}$ for the two-section impedance transformer (30 test points).

We consider the microstrip right-angle bend of Fig.6(a). Design parameters are width $W$, substrate height $H$ and dielectric constant $\varepsilon_{r}$. The region of interest is 20 mil $\leq W \leq$ $30 \mathrm{mil}, 8 \mathrm{mil} \leq H \leq 16 \mathrm{mil}$ and $8 \leq \varepsilon_{r} \leq 10$. The frequency range is $1 \mathrm{GHz}$ to $31 \mathrm{GHz}$. The reference point is $\boldsymbol{x}^{0}=\left[\begin{array}{ll}25 & 12\end{array}\right]^{T}$, the region size $\delta=\left[\begin{array}{lll}5 & 4 & 1\end{array}\right]^{T}$. The fine model is analyzed by Sonnet's em $^{\text {TM }}$ using $1 \mathrm{mil} \times 1$ mil mesh size. The coarse model is shown in Fig. 6(b). Equivalent circuit parameters are calculated from Kirschning, Jansen and Koster [8].

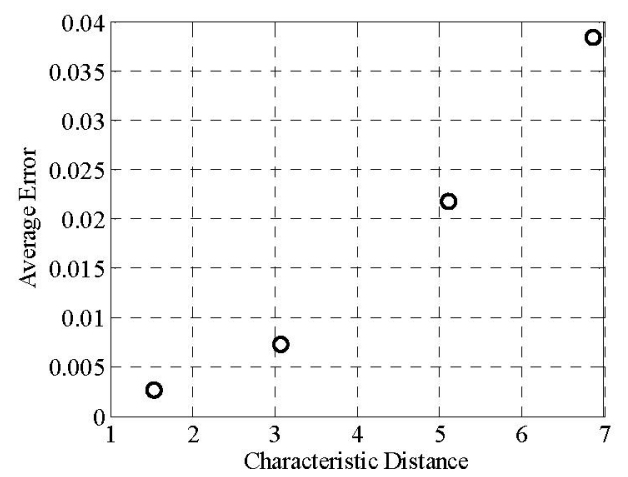

Fig.5. Average modeling error versus characteristic distance $\lambda$ for the twosection impedance transformer. Data for the new surrogate model with base sets $X_{B 1}-X_{B 4}$. Verification for 30 test points.

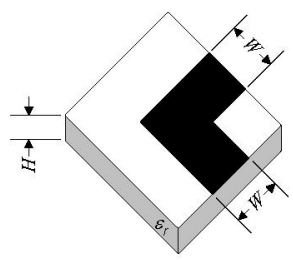

(a)

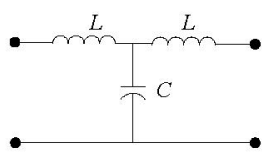

(b)
Fig.6. The microstrip right-angle bend: the fine model (a) and the coarse model (b).

We compared the accuracy of the standard model and the new model for different base sets. Table III shows details of the base sets used in the experiments. Accuracy was tested using 30 random points; the $l_{2}$ norm of the difference between the fine model response and the corresponding surrogate model response was used as an error measure.

Table IV shows numerical results (error statistics) for the standard model and the new surrogate with all the base sets considered. Figs. 7-9 show error plots (modulus of difference between the fine model and the corresponding surrogate model response versus frequency) for the standard model and the new model with base sets $X_{B 3}$ and $X_{B 4}$, respectively. All the experiments were performed using constant $C$ equal to 1.0 (cf. (4)). Fig. 10 shows dependence of average modeling error on the characteristic distance $\lambda$.

Similarly as in the previous example, the new model allows us to significantly improve modeling accuracy (with respect to the standard model) by using a larger number of base points.

TABLE III

Base Set Data For Microstrip Bend Example

\begin{tabular}{clcc}
\hline \hline Base set & \multicolumn{1}{c}{ Description } & Number of points & $\lambda$ \\
\hline$X_{B 1}$ & Uniform mesh $+\boldsymbol{x}^{0}$ & 9 & 3.52 \\
$X_{B 2}$ & Uniform mesh & 27 & 2.44 \\
$X_{B 3}$ & Uniform mesh & 64 & 1.83 \\
$X_{B 4}$ & Uniform mesh & 125 & 1.47 \\
\hline \hline
\end{tabular}




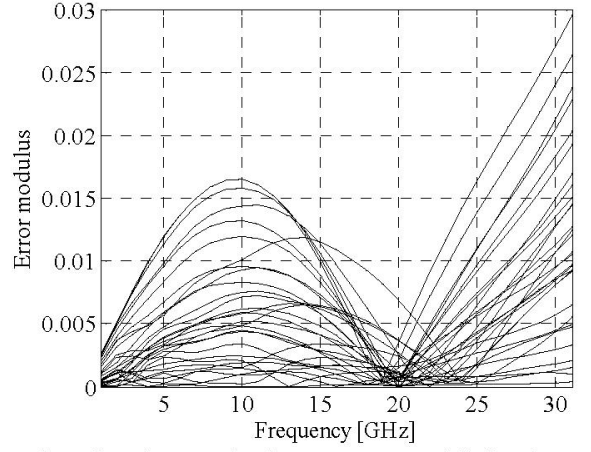

Fig.7. Error plots for the standard surrogate model for the microstrip rightangle bend ( 30 test points)

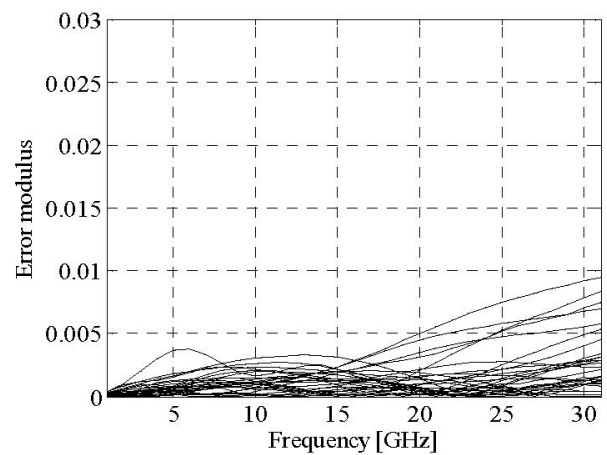

Fig.8. Error plots for the new surrogate model with base set $X_{B 3}$ for the microstrip right-angle bend ( 30 test points).

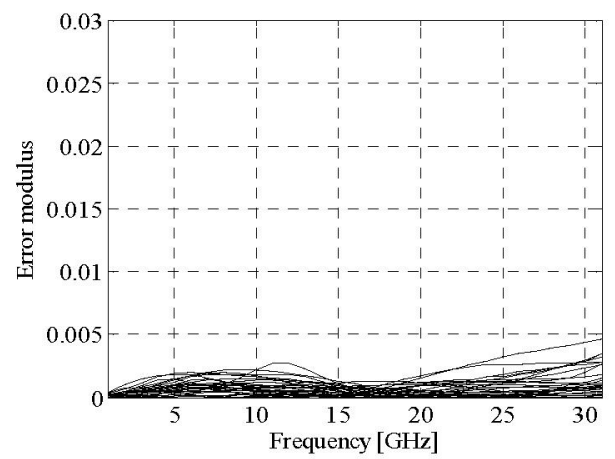

Fig.9. Error plots for the new surrogate model with base set $X_{B 4}$ for the microstrip right-angle bend ( 30 test points).

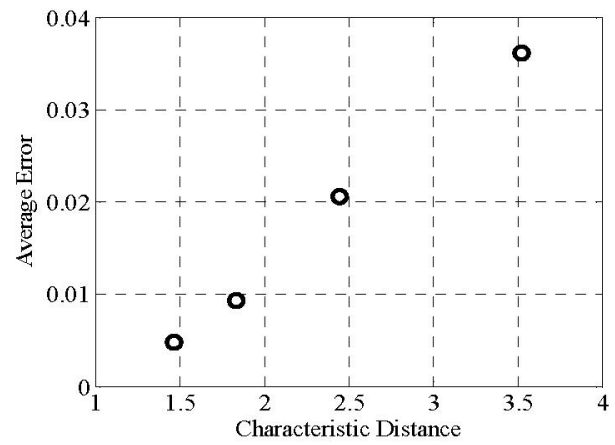

Fig.10. Average modeling error versus characteristic distance $\lambda$ for the microstrip right-angle bend. Data for the new surrogate model with base sets $X_{B 1}-X_{B 4}$. Verification for 30 test points.
TABLE IV

MODELING RESUlts FOR For MiCROSTRIP BEND EXAMPLE. VERIFICATION FOR 30 RANDOM TEST POINTS

\begin{tabular}{ccccc}
\hline \hline Model & Base set & $\begin{array}{c}\text { Average } \\
\text { Error }\end{array}$ & $\begin{array}{c}\text { Maximum } \\
\text { Error }\end{array}$ & $\begin{array}{c}\text { Standard } \\
\text { Deviation }\end{array}$ \\
\hline Standard & star dist. & 0.0331 & 0.0825 & 0.0213 \\
New & $X_{B 1}$ & 0.0361 & 0.0826 & 0.0161 \\
New & $X_{B 2}$ & 0.0206 & 0.0473 & 0.0115 \\
New & $X_{B 3}$ & 0.0093 & 0.0279 & 0.0065 \\
New & $X_{B 4}$ & 0.0049 & 0.0122 & 0.0029 \\
\hline \hline
\end{tabular}

\section{CONCLUSIONS}

A new SM-based modeling methodology is presented. It utilizes parameter extraction with variable weight coefficients. This concept allows the base points that are closer to the model evaluation point to have a bigger impact on the surrogate response. As a result, the new methodology can efficiently handle any base set and has the property of increasing modeling accuracy when the number of base points increases. Examples show robustness of the proposed method.

\section{REFERENCES}

[1] J.W. Bandler, R.M. Biernacki, S.H. Chen, P.A. Grobelny and R.H. Hemmers, "Space mapping technique for electromagnetic optimization," IEEE Trans. Microwave Theory Tech., vol. 42, Dec. 1994, pp. 2536-2544.

[2] J.W. Bandler, R.M. Biernacki, S.H. Chen, R.H. Hemmers and K. Madsen, "Electromagnetic optimization exploiting aggressive space mapping," IEEE Trans. Microwave Theory Tech., vol. 43, Dec. 1995 , pp. 2874-2882.

[3] M.H. Bakr, J.W. Bandler and N. Georgieva, "Modeling of microwave circuits exploiting space derivative mapping," IEEE MTT-S Int. Microwave Symp. Dig., Anaheim, CA, June 1999, pp. 715-718.

[4] J.W. Bandler, N. Georgieva, M.A. Ismail, J.E. Rayas-Sánchez and Q. J. Zhang, "A generalized space mapping tableau approach to device modeling," IEEE Trans. Microwave Theory Tech., vol. 49, Jan. 2001, pp. 67-79.

[5] J.W. Bandler, M.A. Ismail, J.E. Rayas-Sánchez and Q.J. Zhang, "Neuromodeling of microwave circuits exploiting space mapping technology," IEEE Trans. Microwave Theory Tech., vol. 47, Dec. 1999, pp. 2417-2427.

[6] S. Koziel, J.W. Bandler, A.S. Mohamed and K. Madsen, "Enhanced surrogate models for statistical design exploiting space mapping technology," IEEE MTT-S Int. Microwave Symp. Dig., Long Beach, CA, June 2005, pp. 1609-1612.

[7] J.W. Bandler, Q.S. Cheng and S. Koziel, "Implementable space mapping approach to enhancement of microwave device models," IEEE MTT-S Int. Microwave Symp. Dig., Long Beach, CA, June 2005, pp. 1139-1142.

[8] M. Kirschning, R. H. Jansen and N. H. L. Koster, "Measurement and computer-aided modeling of microstrip discontinuities by an improved resonator method," IEEE MTT-S Int. Microwave Symp. Dig., Boston, MA, May 1983, pp. 495-497. 